\title{
The Impact of Large-scale Renewable Integration on Europe's Energy Corridors (2030 - 2050)
}

\author{
Jeroen de Joode (ECN)
}

Other contributors: Gianluigi Migliavacca, Alessandro Zani, Andrea Grassi, Angelo l'Abbate (RSE), Karina Veum, Ozge Ozdemir, Adriaan van der Welle (ECN)

$34^{\text {th }}$ IAEE international Conference Stockholm, 20 ${ }^{\text {th }}$ June 2011 


\section{Outline}

1. Introduction \& focus

2. Approach

3. Results

4. Conclusions

5. Recommendations 


\section{Introduction \& focus}

- Increasing deployment of low carbon energy producing technologies

- Affects markets and infrastructure requirements

- Integral assessment of electricity and gas markets

- SUSPLAN project

- Development of regional and pan-European guidelines for more efficient integration of renewable energy into future infrastructures

- Focus presentation:

- What are infrastructure developments in different futures?

- What is the impact of increasing RES on the energy system? 


\section{Approach}

Model-based

- Simulation model representing European electricity market and transmission infrastructure (MTSIM model RSE)

- Simulation model representing European gas market and gas infrastructure (transmission, LNG, storage) (Gastale model ECN)

- Economic optimization

- Interactive analysis

- Iterations between the two models, both allowing for optimal usage of existing capacity and expansion of capacity

- Long-term perspective

- Starting point 2030, analysis for 2030 - 2050

- Storyline-based

- See next slide 


\section{Approach Introduction to SUSPLAN Storylines}

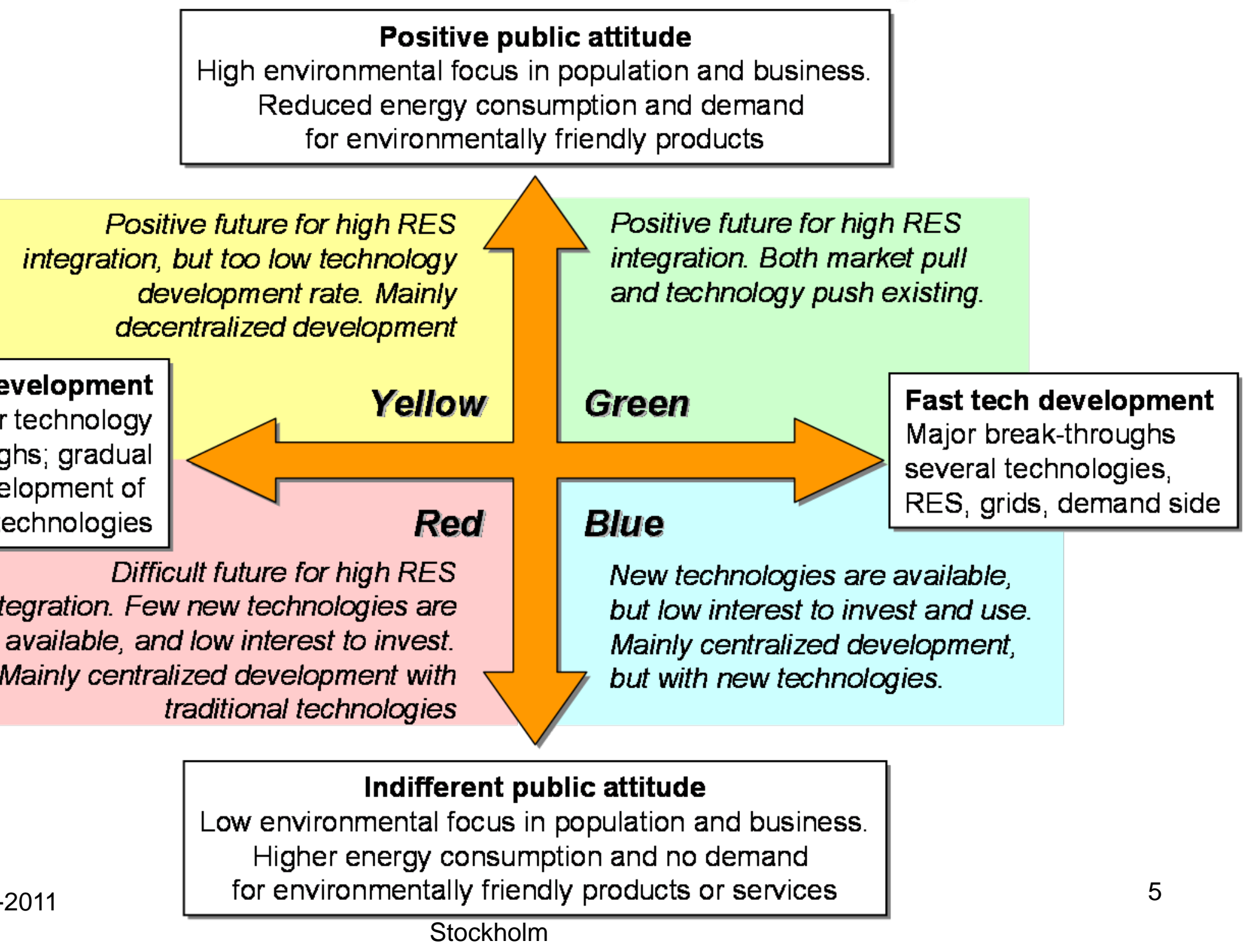

\section{Slow tech development}

No major technology break-throughs; gradual development of current technologies
Difficult future for high RES integration. Few new technologies are available, and low interest to invest. Mainly centralized development with traditional technologies 


\section{Results}




\section{Reduction in $\mathrm{CO}_{2}$ emissions across storylines}

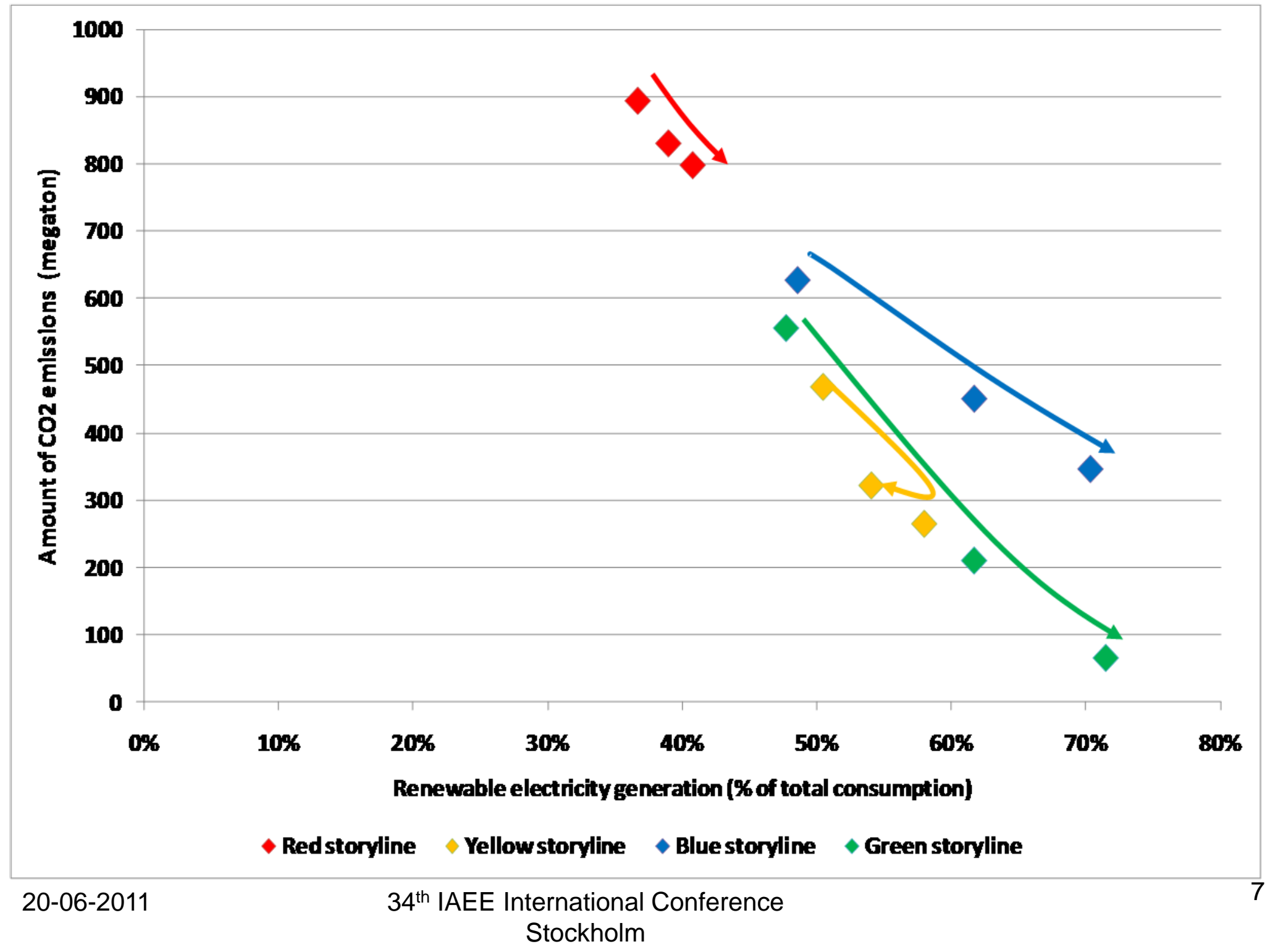




\section{Electricity generation cost}

\section{Contrast between capital \& operational cost impact}

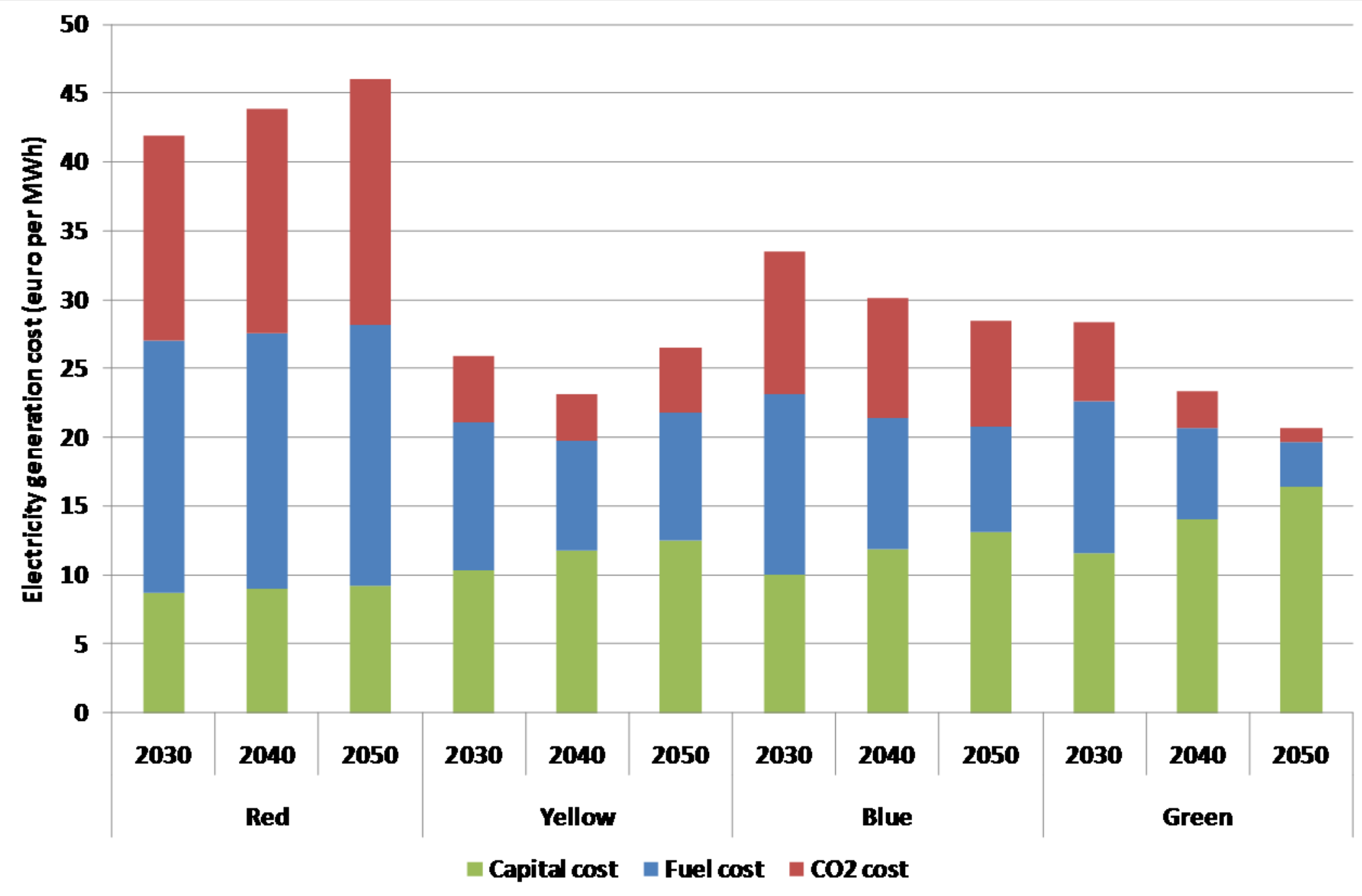




\section{Electricity corridors}
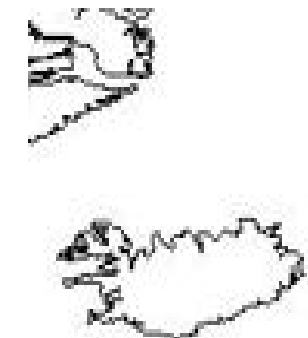
storylines:

DC corridors connecting Northern Europe and UK with Central Europe

- AC corridors connecting Central Europe with Iberian Peninsula

DC/AC corridors in Eastern and South-Eastern Europe

DC corridor from NorthAfrica to Southern Europe

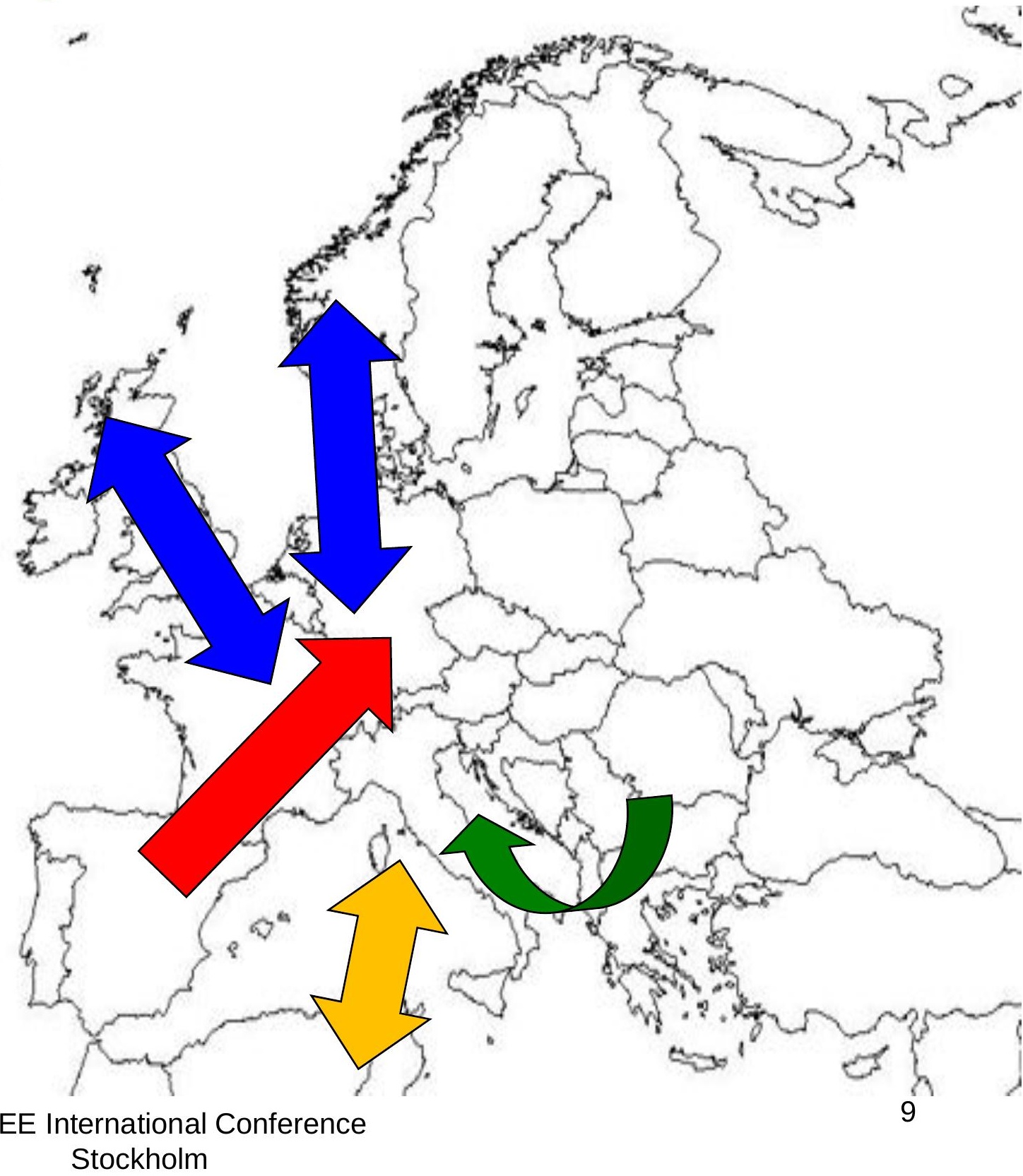




\section{Total gas consumption}

\section{Power sector is main driver across storylines}

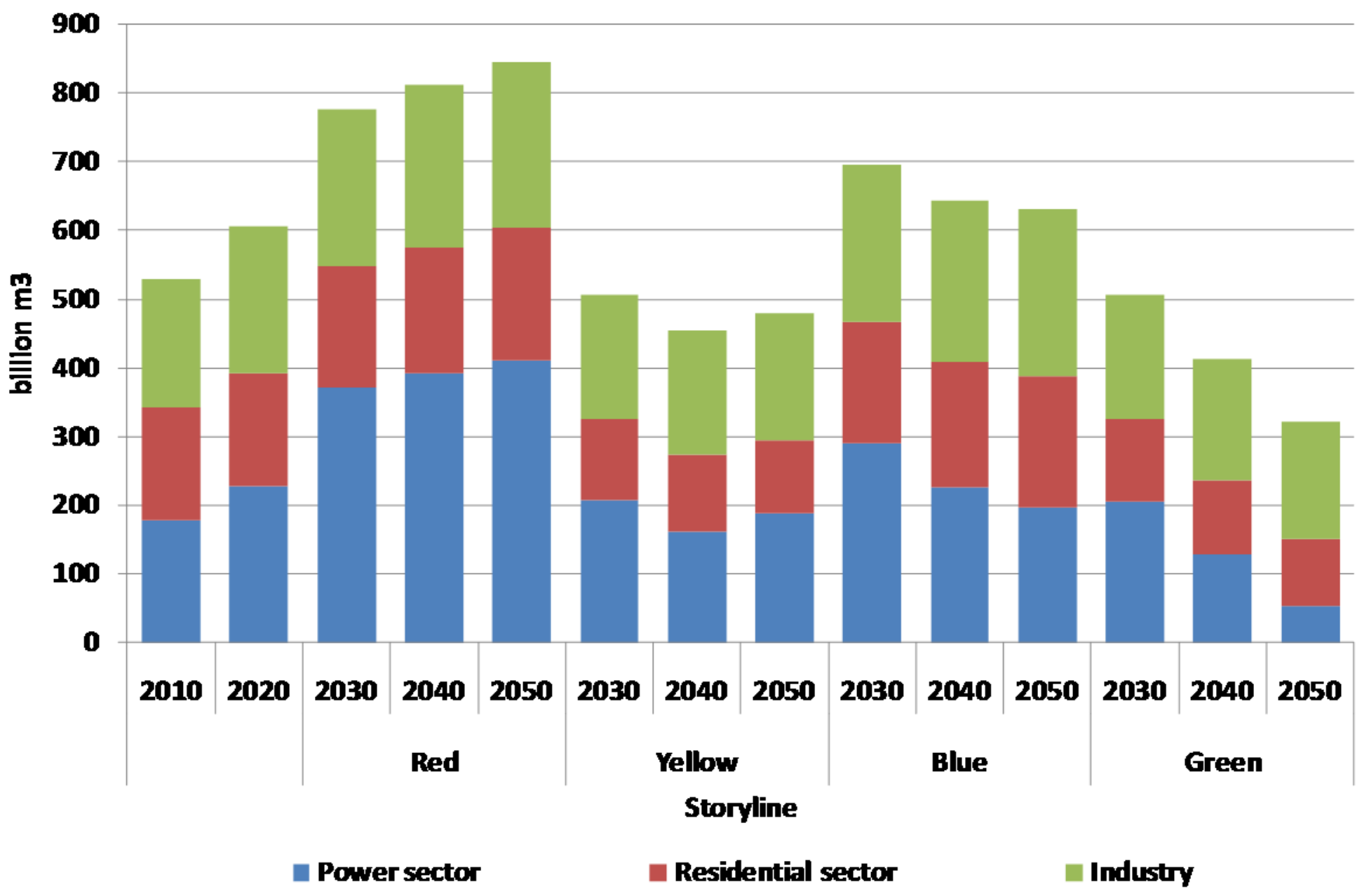


Change in gas demand $2010-2050$

\section{(billion m3)}

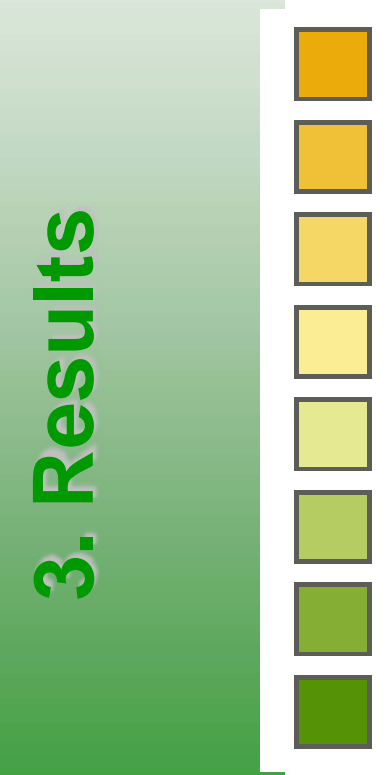

-69 to -50
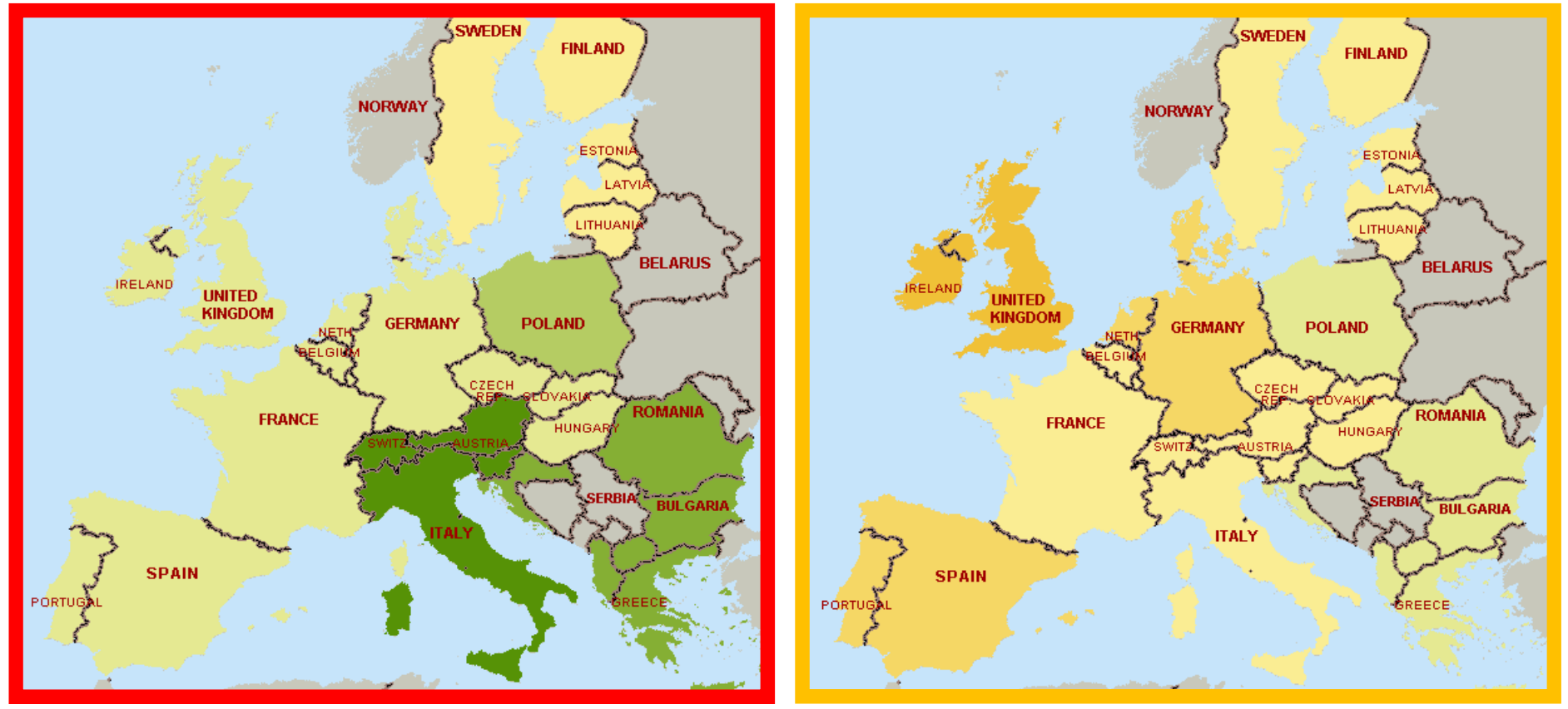

-49 to -30

-29 to -10

-9 to 10

11 to 30

31 to 50

51 to 70

71 to 90

20-06-2011
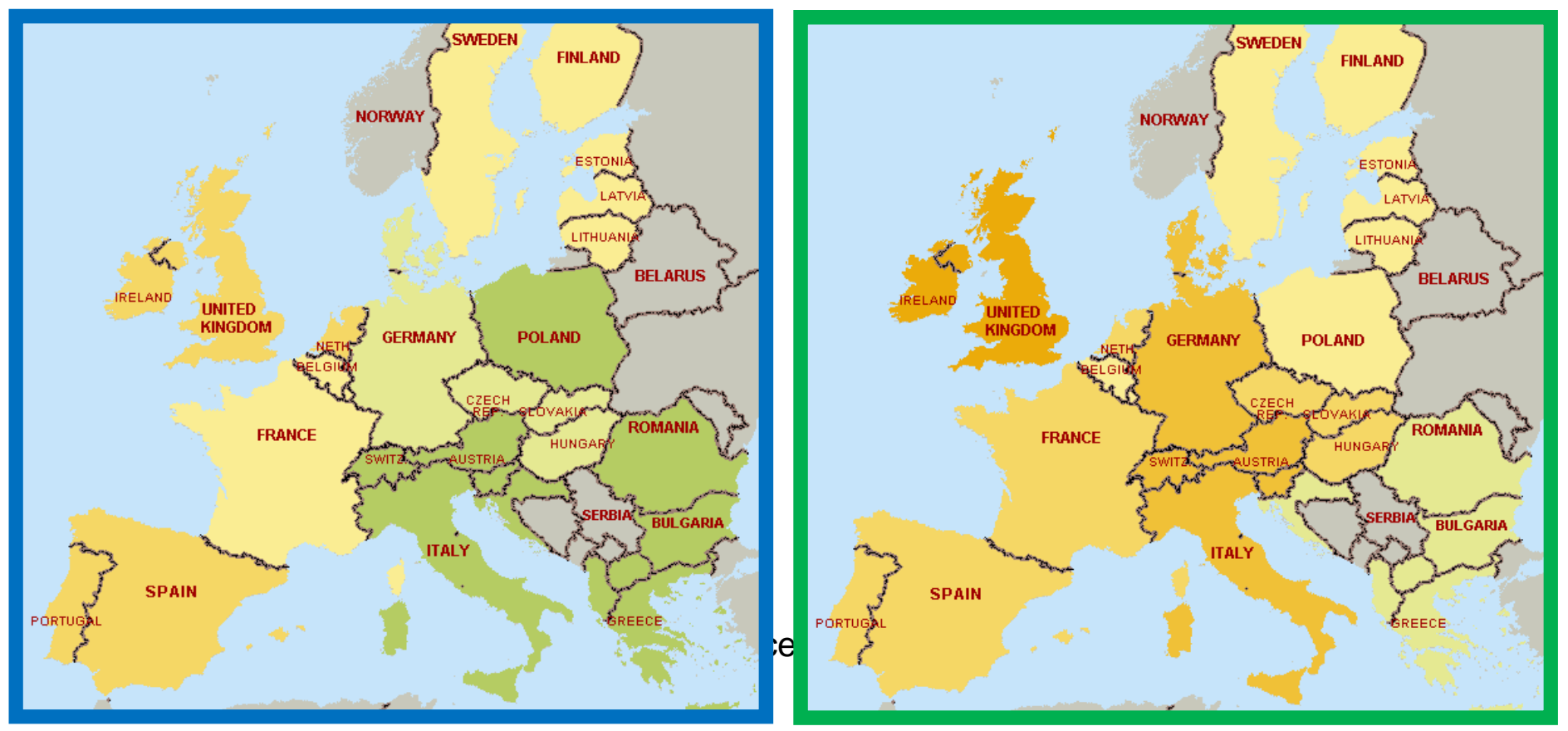


\section{EU gas supply sources}

$\frac{n}{\frac{D}{3}}$

mं
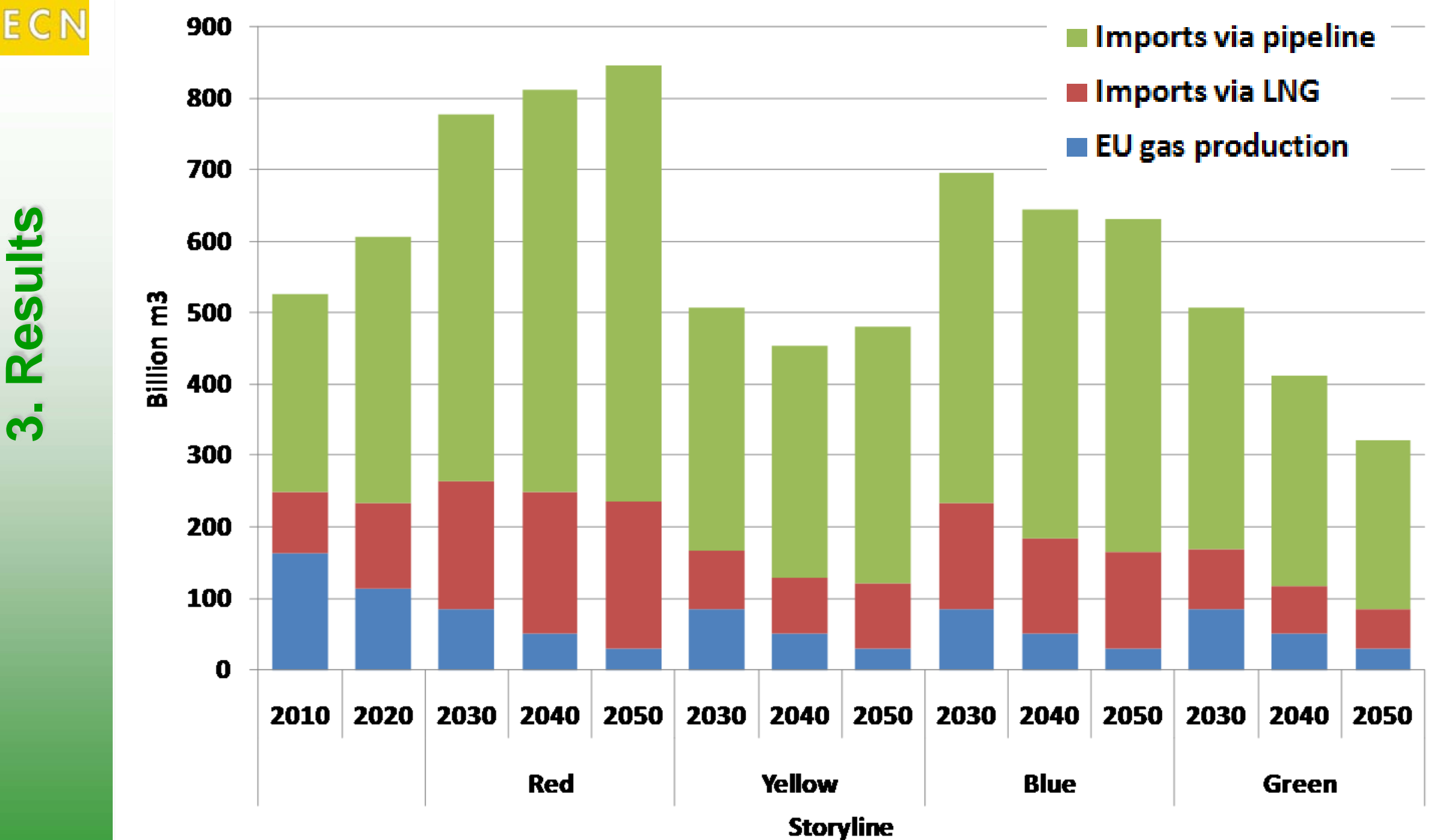


\section{Main observations on gas corridors and hubs}

- Turkey - Southeastern Europe strong corridor in all storylines

- Including downstream expansions in region

Depletion of gas reserves in the UK and the Netherlands

- Limited infra expansion in Northwestern Europe

- Most important storyline differences occur in South and Southwestern Europe

- Pipeline imports North Africa - Spain / Italy

- LNG imports in Italy \& Spain

- Italy as gas hub in high demand storylines, triggering pipeline expansion downstream (region) 


\section{Summary across storylines}

\section{Yellow}

\begin{tabular}{|l|l|rrr|}
\hline & & 2030 & 2040 & 2050 \\
\hline Electricity consumption & PWh & 4.2 & 4.2 & 4.2 \\
\hline Renewable electricity generation & PWh & 2.1 & 2.4 & 2.3 \\
\hline Gas consumption & $\%$ & $50 \%$ & $58 \%$ & $54 \%$ \\
\hline Gas imports & Billion m3 & 507 & 455 & 480 \\
\hline Electricity infrastructure expansion & GW & & 55 & 22 \\
\hline Gas pipeline expansion & Billion m3 & 142 & 35 & 41 \\
\hline CO2 emissions electricity sector & Megation & 468 & 264 & 321 \\
\hline
\end{tabular}

\section{Red}

Electricity consumption

Electricity consumption

Renewable electricity generation

$\%$

Gas consumption

Gas imports

Billion $\mathrm{m} 3$

$20302040 \quad 2050$

Electricity infrastructure expansion

Gas pipeline expansion

Billion $\mathrm{m} 3$

$\begin{array}{lll}4.8 & 5.1 & 5.3\end{array}$

PWh

$\mathrm{PWh}$

$\%$

$\begin{array}{lll}1.8 & 2.0 & 2.2\end{array}$

$37 \% \quad 39 \% \quad 41 \%$

$777 \quad 812 \quad 846$

$692 \quad 761 \quad 815$

GW

Billion $\mathrm{m} 3$

Megaton
$36 \quad 20$

$\begin{array}{lll}250 & 93 \quad 125\end{array}$

$893 \quad 830 \quad 798$

\begin{tabular}{|c|c|c|c|c|}
\hline & & 2030 & 2040 & 2050 \\
\hline Electricity consumption & PWh & 4.2 & 4.2 & 4.2 \\
\hline \multirow[t]{2}{*}{ Renewable electricity generation } & $\mathrm{PWh}$ & 2.0 & 2.6 & 3.0 \\
\hline & $\%$ & $48 \%$ & $62 \%$ & $71 \%$ \\
\hline Gas consumption & Billion m3 & 507 & 412 & 321 \\
\hline Gas imports & Billion m3 & 422 & 361 & 291 \\
\hline Electricity infrastructure expansion & $\mathrm{GW}$ & & 72 & 65 \\
\hline Gas pipeline expansion & Billion m3 & 143 & 28 & 34 \\
\hline CO2 emissions electricity sector & Megaton & 556 & 210 & 66 \\
\hline \multicolumn{5}{|l|}{ Blue } \\
\hline & & 2030 & 2040 & 2050 \\
\hline Electricity consumption & PWh & 4.9 & 5.1 & 5.4 \\
\hline \multirow[t]{2}{*}{ Renewable electricity generation } & $\mathrm{PWh}$ & 2.4 & 3.2 & 3.8 \\
\hline & $\%$ & $49 \%$ & $62 \%$ & $70 \%$ \\
\hline Gas consumption & Billion $\mathrm{m} 3$ & 696 & 644 & 631 \\
\hline Gas imports & Billion m3 & 611 & 593 & 601 \\
\hline Electricity infrastructure expansion & GW & & 56 & 56 \\
\hline Gas pipeline expansion & Billion m3 & 271 & 50 & 48 \\
\hline CO2 emissions electricity sector & Megaton & 627 & 451 & 347 \\
\hline
\end{tabular}

\section{Stockholm}




\section{Summary across storylines Electricity consumption - infra expansion}

\begin{tabular}{|c|c|c|c|c|}
\hline \multicolumn{5}{|l|}{ Yellow } \\
\hline & & 2030 & 2040 & 2050 \\
\hline Electricity consumption & PWh & 4.2 & 4.2 & 4.2 \\
\hline \multirow[t]{2}{*}{ Renewable electricity generation } & PWh & 2.1 & 2.4 & 2.3 \\
\hline & $\%$ & $50 \%$ & $58 \%$ & $54 \%$ \\
\hline Gas consumption & Billion $\mathrm{m} 3$ & 507 & 455 & 480 \\
\hline Gas imports & Billion $\mathrm{m} 3$ & 422 & 403 & 449 \\
\hline Electricity infrastructure expansion & $\mathrm{GW}$ & & 55 & 22 \\
\hline Gas pipeline expansion & Billion $\mathrm{m} 3$ & 142 & 35 & 41 \\
\hline CO2 emissions electricity sector & Megaton & 468 & 264 & 321 \\
\hline
\end{tabular}

Red

Electricity consumption

Renewable electricity generation

Gas consumption

Gas imports

Electricity infrastructure expansion

Gas pipeline expansion

CO2 emissions electricity sector

$20302040 \quad 2050$

\section{PWh}

PWh

$\begin{array}{rrr}1.8 & 2.0 & 2.2 \\ 37 \% & 30 \% & 41 \%\end{array}$

$\%$

$\begin{array}{llll}\text { Billion } \mathrm{m} 3 & 777 & 812 \quad 846\end{array}$

Billion $m^{3}$

GW

Billion $\mathrm{m} 3$

$602 \quad 76$

\section{Green}

\begin{tabular}{|c|c|c|c|c|}
\hline & & 2030 & 2040 & 20 \\
\hline Electricity consumption & PWh & 4.2 & 4.2 & \\
\hline \multirow[t]{2}{*}{ Renewable electricity generation } & PWh & 2.0 & 2.0 & 3.0 \\
\hline & $\%$ & $48 \%$ & $62 \%$ & $71 \%$ \\
\hline Gas consumption & Billion $\mathrm{m} 3$ & 507 & 412 & 321 \\
\hline Gas imports & Billion & 422 & 361 & 291 \\
\hline Electricity infrastructure expansion & GW & & 72 & \\
\hline Gas pipeline expansion & Billion $\mathrm{m}$ & 145 & 28 & 32 \\
\hline $\mathrm{CO} 2$ emissions electricity sector & Megaton & 556 & 210 & 66 \\
\hline
\end{tabular}

Blue

\begin{tabular}{lll|l}
4.8 & 5.1 & 5.3 \\
\hline
\end{tabular}

lectricity consumption

Renewable electricity generation

PWh
PWh
$\%$

$2030 \quad 2040 \quad 2050$

$37 \% \quad 39 \% \quad 41 \%$

Gas consumption

Billion $\mathrm{m} 3$

Billion $\mathrm{m}_{3}$

\begin{tabular}{|r|r|r|}
\hline 4.9 & 5.1 & 5.4 \\
2.4 & 3.2 & 3.8 \\
$49 \%$ & $62 \%$ & $70 \%$ \\
\hline 696 & 644 & 631 \\
611 & 593 & 601 \\
& 56 & 56 \\
\hline 271 & 50 & 48 \\
\hline 627 & 451 & 347 \\
\hline
\end{tabular}

\section{0}

Electricity infrastructure expansion

Gas pipeline expansion

$\mathrm{CO} 2$ emissions electricity sector
GW

Billion $\mathrm{m} 3$

Megaton 


\section{Conclusions}

- Increasing RES has large consequences for investment requirements in trans-national electricity infrastructure (AC and DC)

- If comparative advantages in RES potential in Europe are used

- Results in particular electricity corridors

- Power sector main driver for gas market developments

- Differences across Europe $\rightarrow$ corridors and LNG hubs

- Increase in RES has positive impact on operational costs, but negative impact on capital costs, net impact is likely to be positive with increasing RES share.

- Higher electricity infrastructure requirements may partly be compensated by lower gas infrastructure requirements 


\section{Recommendations}

Further support for focused infrastructure policy, with particular attention for some corridors (i.e. EU infrastructure package)

Need for adequate policy signals regarding long-term $\mathrm{CO} 2$ price, since it is a major uncertainty in shaping of electricity generation mix

- Electricity infrastructure affects gas infrastructure (and vice versa), so energy policy should not focus on one sector in isolation

- What is the future role of gas in the EU energy mix? 